\title{
Hydrodynamic excitations of trapped dipolar fermions
}

\author{
Krzysztof Góral, ${ }^{1}$ Mirosław Brewczyk, ${ }^{2}$ and Kazimierz Rzążewski ${ }^{1}$ \\ ${ }^{1}$ Center for Theoretical Physics, Polish Academy of Sciences, Aleja Lotników 32/46, 02-668 Warsaw, Poland \\ ${ }^{2}$ Uniwersytet $w$ Biatymstoku, ul. Lipowa 41, 15-424 Biatystok, Poland
}

\begin{abstract}
A single-component Fermi gas of polarized dipolar particles in a harmonic trap can undergo a mechanical collapse due to the attractive part of the dipole-dipole interaction. This phenomenon can be conveniently manipulated by the shape of the external trapping potential. We investigate the signatures of the instability by studying the spectrum of low-lying collective excitations of the system in the hydrodynamic regime. To this end, we employ a time-dependent variational method as well as exact numerical solutions of the hydrodynamic equations of the system.
\end{abstract}

PACS numbers: 03.75.Ss,05.30.Fk

Following a spectacular series of experimental achievements with Bose-Einstein condensates in dilute trapped atomic gases (for a review see, e.g., [1, 2]), dilute gaseous atomic Fermi systems have drawn intensified interest only very recently. After the first successful realization of quantum degeneracy in a potassium gas [3], several other groups have cooled fermionic ${ }^{6} \mathrm{Li}$ below the Fermi temperature and trapped it magnetically or optically [4, 5, 6]. Very recently, two-species Fermi-Bose mixtures have been also achieved in ${ }^{6} \mathrm{Li}-{ }^{23} \mathrm{Na}[7]$ and in ${ }^{40} \mathrm{~K}-{ }^{87} \mathrm{Rb}[8]$. Much emphasis has been put on the attainment of the transition to a superfluid Cooperpaired state [9, 10, 11].

In a single-component Fermi gas at very low energies swave scattering is excluded by the statistics. Therefore, such a system behaves as a nearly ideal one. However, in this situation other types of interactions, usually negligible, come into play. A good candidate is a dipole-dipole force. Its effects might be seen in the behavior of atoms with large permanent magnetic moments (such as chromium [12, 13]) and should be evident in the case of heteronuclear molecules possessing permanent electric dipoles (an example is $\mathrm{ND}_{3}$, which has been cooled in a Stark decelerator down to the millikelvin range [14]). Another option is to induce electric dipoles with the help of strong DC fields [15] or by optical admixing of large dipole moments of Rydberg states [16].

The ground state of a harmonically trapped polarized single-component dipolar Fermi gas in the normal phase has been investigated in [17]. An interesting possibility of mechanical instability of the system has been investigated in detail. Using the Thomas-Fermi theory (together with the Dirac correction) the authors have shown that when the critical values of the total particle number $N$ or of the dipole moment $d$ are reached, the system collapses (for an analogous discussion of a dipolar Bose condensate see [16]). It has been shown that this behavior is governed by a parameter $N^{\frac{1}{6}} \varepsilon$, where

$$
\varepsilon=\left(\omega m^{3} / \hbar^{5}\right)^{\frac{1}{2}} d^{2} .
$$

In Eq. (1) $\omega$ is the transverse frequency of an axially symmetric harmonic trap (the trap axis being parallel to the dipole polarization) and $m$ is the particle mass. The critical value of $N^{\frac{1}{6}} \varepsilon$ depends on the trap aspect ratio $\beta=\omega_{z} / \omega$ ( $\omega_{z}$ is the axial frequency of the trap). A detailed stability diagram is presented in Fig.1 of Ref.[17]. Here we summarize its main features pertinent to the following discussion. The most striking finding is the existence of a characteristic value of the aspect ratio $\beta \simeq 5.2$ above which (i.e., in sufficiently oblate traps) the system is always stable, for any number of particles and any dipole moment. Away from this region the critical values of $N^{\frac{1}{6}} \varepsilon$, above which the system is unstable, tend to lie between 2 and 10 . Generally, the critical $N^{\frac{1}{6}} \varepsilon$ is smaller for traps elongated in the direction of polarization, i.e., for $\beta<1$ (with an exception region for extremely stiff traps with $\beta \ll 1$, where the critical value of $N^{\frac{1}{6}} \varepsilon$ grows again). As regards the experimental prospects of observing the predicted instability, one can say that it will definitely not affect weak magnetic dipoles [12, 13]. However, polar molecules and strong electrically induced atomic dipoles may very well be located in the vicinity of the unstable region.

At this point, a discussion of $\mathrm{p}$-wave induced collapse in a single-component Fermi gas should also be mentioned [18]. Another interesting aspect of the physics of trapped dipolar fermions, an issue of superfluid pairing induced by the dipole-dipole interactions, has also been addressed recently in Refs. 19, 20].

The issue of collective excitations of degenerate Fermi gases is important and its studies have the same motivations as in the case of Bose condensates - the spectrum of collective excitations determines the system dynamics in the lowenergy regime. This way, the analysis of the excitations brings yet another important piece of information about quantum degenerate fermionic gases. A few works have been concerned with the problem. Amoruso et al. [21] as well as Bruun and Clark [22] have investigated hydrodynamic excitations in non-interacting Fermi gases, mainly in spherically symmetric traps. Their work has been extended to the case of anisotropic trapping potentials by Csordás and Graham [23]. Some experimental work should be mentioned in the context of collective excitations - DeMarco and Jin have investigated spin excitations in a fermionic gas of atoms [24].

In the case of degenerate bosons, the spectrum of the collective excitations strongly depends on the particle interactions. In the mean-field picture, this amounts to the dependence on 
both the number of particles and the scattering length. As single-component degenerate fermions at low energies are virtually non-interacting, the problem of interactions is less important and appears in two contexts only: either one deals with a two-component gas (where inter-component scattering takes place) [25] or, in the single-component case, one takes into account dipole-dipole forces. In this paper we study the latter case.

In the hydrodynamic regime, i.e., when collisions ensure local equilibrium, the dynamics of a Fermi gas is governed by the hydrodynamic equations for the fields of velocity $\mathbf{v}(\mathbf{r}, t)$ and density $n(\mathbf{r}, t)$ :

$$
\begin{aligned}
& \frac{\partial n(\mathbf{r}, t)}{\partial t}=-\nabla \cdot[n(\mathbf{r}, t) \mathbf{v}(\mathbf{r}, t)] \\
& \frac{\partial \mathbf{v}(\mathbf{r}, t)}{\partial t}=-\nabla\left[\frac{\hbar^{2}}{2 m^{2}}\left[6 \pi^{2} n(\mathbf{r}, t)\right]^{2 / 3}+\frac{1}{2} \mathbf{v}^{2}(\mathbf{r}, t)\right. \\
& \left.+\frac{1}{2} \omega^{2}\left[x^{2}+y^{2}+(\beta z)^{2}\right]+\frac{1}{m} \int d \mathbf{r}^{\prime} n(\mathbf{r}, t) V_{\mathrm{dd}}\left(\mathbf{r}-\mathbf{r}^{\prime}\right)\right]
\end{aligned}
$$

where $V_{\mathrm{dd}}(\mathbf{r})$ is the dipole-dipole potential

$$
V_{\mathrm{dd}}(\mathbf{r})=\frac{d^{2}}{r^{3}}-3 \frac{(\mathbf{d} \cdot \mathbf{r})^{2}}{r^{5}}
$$

In the second of Eqs. 2 one can recognize various components of the Thomas-Fermi formulation for trapped dipolar fermions [17]: the first term on the right-hand side is the quantum pressure (microscopic kinetic energy) resulting from Fermi statistics; the next term describes the macroscopic part of the kinetic energy; the first term in the last line is the harmonic trapping potential and the last term in this line is the dipole-dipole interaction energy.

In order to study low-lying collective excitations, in the following we propose to merge the variational approach (used to find the ground state of a dipolar Fermi gas in Ref.[17]) with the time-dependent variational method used to study bosonic oscillations (see, e.g., Refs. [26, 27, 28]). A related approach has been developed by Zaremba and Tso [29] to model a parabolically confined electron gas.

First, we introduce the Lagrangian density

$$
\begin{aligned}
& \mathcal{L}(\mathbf{r}, t)=-\hbar n(\mathbf{r}, t) \frac{\partial \chi(\mathbf{r}, t)}{\partial t}-\frac{\hbar^{2}}{m} \frac{1}{20 \pi^{2}}\left[6 \pi^{2} n(\mathbf{r}, t)\right]^{5 / 3} \\
& -\frac{\hbar^{2}}{2 m} n(\mathbf{r}, t)(\nabla \chi(\mathbf{r}, t))^{2}-\frac{1}{2} m \omega^{2}\left[x^{2}+y^{2}+(\beta z)^{2}\right] n(\mathbf{r}, t) \\
& -\frac{1}{2} \int d \mathbf{r}^{\prime} n(\mathbf{r}, t) V_{\mathrm{dd}}\left(\mathbf{r}-\mathbf{r}^{\prime}\right) n\left(\mathbf{r}^{\prime}, t\right)
\end{aligned}
$$

where $\mathbf{v}(\mathbf{r}, t)=(\hbar / 2 m) \lim _{\vec{r}_{1} \rightarrow \vec{r}_{2}}\left(\vec{\nabla}_{1}-\vec{\nabla}_{2}\right) \chi\left(\vec{r}_{1}, \vec{r}_{2}, t\right)$ and $n(\mathbf{r}, t)$ is the spatial one-particle density. $\chi$ is usually termed the potential of the velocity field. The Lagrangian density $\mathcal{L}(\mathbf{r}, t)$ is chosen such that the Lagrange equations for the conjugate variables $n$ and $\chi$, following from the Lagrangian $L=$ $\int d \mathbf{r} \mathcal{L}(\mathbf{r}, t)$, reproduce the hydrodynamic equations (2) for the Fermi gas.

From this point we proceed in a close analogy to the procedure employed for bosons in Ref.[28]. We introduce a Gaussian variational ansatz for the density $n(\mathbf{r}, t)$ :

$$
n(x, y, z, t)=\frac{N}{\pi^{3 / 2} w_{x}(t) w_{y}(t) w_{z}(t)} \prod_{\eta=x, y, z} e^{-\left[\eta-\eta_{0}(t)\right]^{2} / w_{\eta}^{2}(t)},
$$

where $w_{\eta}(t)$ are time-dependent widths, and a variational form for the phase:

$$
\chi(\mathbf{r}, t)=\sum_{\eta=x, y, z}\left[\eta \alpha_{\eta}(t)+\eta^{2} \beta_{\eta}(t)\right]
$$

where $\alpha_{\eta}(t)$ and $\beta_{\eta}(t)$ are variational parameters - see also $[26,27]$. The linear part of the phase ansatz describes the drift of the Fermi fluid (a constant part of the velocity field) whereas the quadratic term corresponds to oscillations (a linear part of the velocity). This kind of ansatz has proved to work very well in reproducing the static properties of trapped dipolar fermions [17]. It was also used successfully to model the dynamical behavior of trapped dipolar Bose-Einstein condensates [27, 28]. This way, the problem is reduced to the study of a few Lagrange equations for the variational parameters $w_{\eta}(t), \eta_{0}(t), \alpha_{\eta}(t)$, and $\beta_{\eta}(t)$ :

$$
\begin{aligned}
& \ddot{w}_{\eta}=c \frac{\hbar^{2}}{m^{2}} \frac{1}{w_{\eta}}\left[\frac{N}{w_{x} w_{y} w_{z}}\right]^{2 / 3}-\omega_{\eta}^{2} w_{\eta}+\frac{2}{N m} \frac{\partial I}{\partial w_{\eta}} \\
& \ddot{\eta}_{0}+\omega_{\eta}^{2} \eta_{0}=0 \\
& \alpha_{\eta}=\frac{m}{\hbar}\left[\dot{\eta}_{0}-\frac{\eta_{0} \dot{w}_{\eta}}{w_{\eta}}\right] \\
& \beta_{\eta}=\frac{m}{2 \hbar} \frac{\dot{w}_{\eta}}{w_{\eta}}
\end{aligned}
$$

where $c=6^{5 / 3} \sqrt{3 / 5} \pi^{1 / 3} / 25$ is a numerical factor and

$$
I=-\frac{1}{2} \int d \mathbf{r} d \mathbf{r}^{\prime} n(\mathbf{r}, t) V_{\mathrm{dd}}\left(\mathbf{r}-\mathbf{r}^{\prime}\right) n\left(\mathbf{r}^{\prime}, t\right) .
$$

Again, we can recognize various terms of the Thomas-Fermi theory in Eqs. (710): the first term of the right-hand side of Eq.(7) corresponds to the quantum pressure, the second term comes from the trapping potential, and the last term results from the dipole-dipole interaction energy.

As we see from Eq. [8, the center-of-mass motion (described by $\left.\eta_{0}(t)\right)$ is decoupled and follows simple harmonic oscillations, just as in the case of bosons [26, 28]. The only independent variables are the widths $w_{\eta}(t)$. Their dynamics, governed by Eq.(7), can be viewed as a motion of a fictitious particle in the following potential:

$$
U\left(w_{x}, w_{y}, w_{z}\right)=\frac{1}{2} \sum_{\eta} \omega_{\eta}^{2} w_{\eta}^{2}+c \frac{3 \hbar^{2}}{2 m^{2}}\left[\frac{N}{w_{x} w_{y} w_{z}}\right]^{2 / 3}-\frac{2 I}{N m}
$$




\begin{tabular}{|c|c|c|c|}
\hline aspect ratio $\beta$ & $\beta>0.42$ & \multicolumn{2}{|c|}{$\beta \leq 0.42$} \\
\hline lowest mode & quadrupole (unstable for $\beta<5.2)$ & $N^{\frac{1}{6}} \varepsilon$ below the second crossing & quadrupole \\
\cline { 3 - 4 } & & $N^{\frac{1}{6}} \varepsilon$ above the second crossing & breathing (unstable) \\
\hline
\end{tabular}

TABLE I: Lowest and unstable modes for harmonically trapped dipolar fermions.

By diagonalizing the matrix of second derivatives of this potential (evaluated at the stationary point, i.e., for the ground state values of the widths - as calculated in Ref.[17]) one can obtain the frequencies and the shapes of three low-lying eigenmodes of the cloud. These modes have the same structure as in the bosonic case - see Fig 1

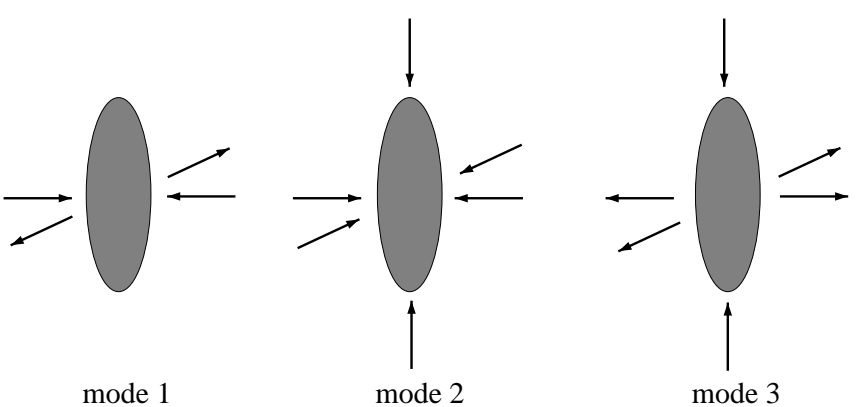

FIG. 1: Graphical representation of oscillation modes given by the variational analysis. We refer to mode 2 as the breathing one and to mode 3 as the quadrupole one. Mode 1 will be called the 2D quadrupole mode.

An interesting question in the analysis of the instability induced by dipole-dipole interactions concerns the identification of the mode that becomes unstable (which is indicated by its frequency approaching zero) at the critical value of the dipolar parameter $N^{\frac{1}{6}} \varepsilon$. We have analyzed this problem for different cylindrically symmetric traps.

Let us first concentrate on cigar-shaped traps $(\beta<1)$. For $0.42<\beta<1$, the lowest mode is always the quadrupole mode (mode 3 in Fig 1) and its frequency goes to zero as the critical value of $N^{\frac{1}{6}} \varepsilon$ is approached. At the characteristic value of $\beta \simeq 0.42$ two avoided crossings are present - see Fig 2 Away from the instability (i.e., for $N^{\frac{1}{6}} \varepsilon<2.40$ ) and below both crossings the breathing mode (mode 2) has the highest frequency. The quadrupole mode is the lowest one and mode 1 (2D quadrupole) is the intermediate one. At the first crossing, the nature of two higher modes is exchanged - now mode 1 (2D quadrupole) is the highest one. This first crossing is present in all traps with $\beta<1$. At the second crossing the breathing mode 2 acquires the lowest frequency and becomes unstable. For $N^{\frac{1}{6}} \varepsilon$ slightly below the second crossing, the oscillation of the quadrupole (lowest) mode is almost purely axial (the eigenmode is $(-\delta,+\delta, \sim 1)$, where $\delta$ indicates a very small value). Just above the crossing, the shape of the lowest (breathing) mode remains very axial with no phase difference between the oscillations in the $\mathrm{X}$ and $\mathrm{Y}$ directions (the eigenmode is now $(+\delta,+\delta, 1))$. This is always the case for $\beta \leq 0.42$.
For smaller values of the trap aspect ratio, the positions of the two crossings merge (e.g., for $\beta=0.031$ the crossings are at $N^{\frac{1}{6}} \varepsilon=2.99$, very close to the instability). For pancake traps $(\beta>1)$, the mode that drives the instability (which takes place as long as $\beta<5.2$ ) is always the quadrupole mode. For clarity, the results are summarized in Table $\square$

The time-dependent variational method employed thus far provides approximate solutions of the hydrodynamic equations of the system. To confirm their validity, we have performed exact numerical simulations of the hydrodynamic equations (2). First, we find the ground state of the system by the propagation in imaginary time and use it as an initial condition for the simulations. Then, we perturb the trapping potential for a fraction of a trap period and let the system evolve while monitoring the resultant oscillations. Finally, we extract the frequency of the oscillations and identify the corresponding modes. The results are presented in Fig 2 and agree very well with the variational solutions.

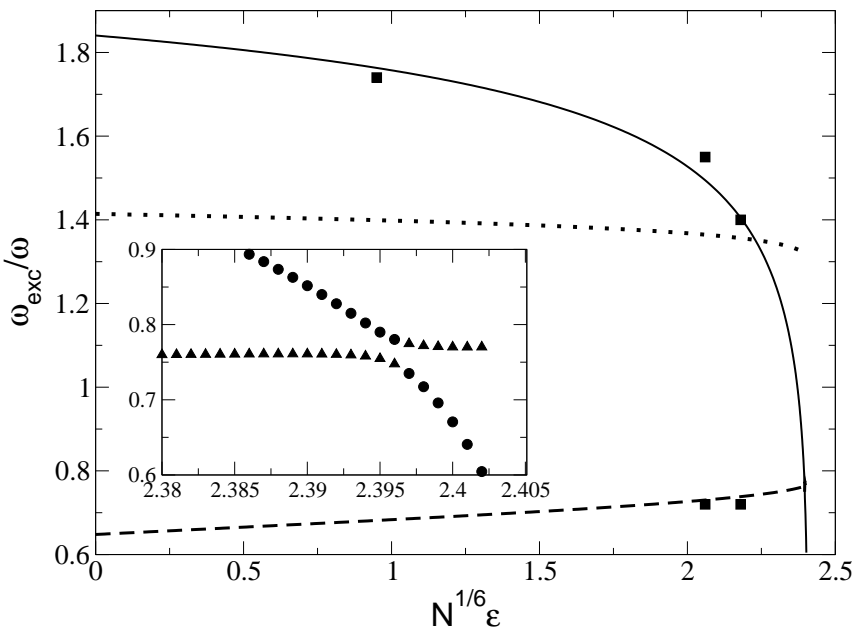

FIG. 2: Excitation frequencies of low-lying modes for the dipolar Fermi gas in a cigar-shaped trap with $\beta=0.42$. The (unstable) mode 2 (breathing) is depicted with a solid line, while mode 1 (2D quadrupole) with a dotted line. Mode 3 (quadrupole) is marked by a dashed line. Lines depict the results of the variational analysis, while squares come from exact numerical solutions of the hydrodynamic equations. Inset shows a magnification of the second avoided crossing (mode 3 denoted by triangles and mode 2 by circles).

To summarize, we have investigated the signatures of the mechanical instability in a single-component Fermi gas of polarized dipolar particles confined in a harmonic trap by studying the spectrum of its low-lying hydrodynamic collective excitations. We have employed a time-dependent variational method, not previously used in the context of excitations of 
dilute atomic Fermi gases, as well as exact solutions of the hydrodynamic equations of the system. We have identified modes driving the instability for various geometries of the trapping potential. In a rough classification, for oblate and slightly prolate traps $(\beta>0.42)$ it is the quadrupole mode that drives the instability, while for cigar-shaped traps with $\beta<0.42$ the breathing mode is an unstable one. Finally, one should note that the presented analysis describes the zerotemperature situation. It would be very interesting, although very challenging, to include the effects of temperature in the treatment.

K.G. acknowledges support by the Polish KBN grant No. 5 P03B 10220 and by the Foundation for Polish Science. Part of the results has been obtained using computers at Interdisciplinary Centre for Mathematical and Computational Modelling of Warsaw University.

[1] J.R. Anglin and W. Ketterle, Nature (London) 416, 211 (2002).

[2] S.L. Rolston and W.D. Phillips, Nature (London) 416, 219 (2002).

[3] B. DeMarco and D.S. Jin, Science 285, 1703 (1999).

[4] A.G. Truscott, K.E. Strecker, W.I. McAlexander, G.B. Partridge, and R.G. Hulet, Science 291, 2570 (2001).

[5] F. Schreck, L. Khaykovich, K.L. Corwin, G. Ferrari, T. Bourdel, J. Cubizolles, and C. Salomon, Phys. Rev. Lett. 87, 080403 (2001).

[6] S.R. Granade, M.E. Gehm, K.M. O'Hara, and J.E. Thomas, Phys. Rev. Lett. 88, 120405 (2002).

[7] Z. Hadzibabic, C.A. Stan, K. Dieckmann, S. Gupta, M.W. Zwierlein, A. Görlitz, and W. Ketterle, Phys. Rev. Lett. 88, 160401 (2002).
[8] G. Roati, F. Riboli, G. Modugno, and M. Inguscio, Phys. Rev. Lett. 89, 150403 (2002).

[9] M. Holland, S.J.J.M.F. Kokkelmans, M.L. Chiofalo, and R. Walser, Phys. Rev. Lett. 87, 120406 (2001).

[10] E. Timmermans, K. Furuya, P.W. Milonni, and A.K. Kerman, Phys. Lett. A 285, 228 (2001).

[11] W. Hofstetter, J.I. Cirac, P. Zoller, E. Demler, and M.D. Lukin, Phys. Rev. Lett. 89, 220407 (2002).

[12] J. Stuhler, P.O. Schmidt, S. Hensler, J. Werner, J. Mlynek, and T. Pfau, Phys. Rev. A 64, 031405 (2001).

[13] J.D. Weinstein, R. deCarvalho, C.I. Hancox, and J.M. Doyle, Phys. Rev. A 65, 021604 (2002).

[14] H.L. Bethlem, G. Berden, F.M.H. Crompvoets, R.T. Jongma, A.J.A. van Roij, and G. Meijer, Nature (London) 406, 491 (2000).

[15] M. Marinescu and L. You, Phys. Rev. Lett. 81, 4596 (1998).

[16] L. Santos, G.V. Shlyapnikov, P. Zoller, and M. Lewenstein, Phys. Rev. Lett. 85, 1791 (2000); 88, 139904(E) (2002).

[17] K. Góral, B.-G. Englert, and K. Rzążewski, Phys. Rev. A 63, 033606 (2001).

[18] R. Roth and H. Feldmeier, Phys. Rev. A 64, 043603 (2001).

[19] M.A. Baranov, M.S. Mar'enko, V.S. Rychkov, and G.V. Shlyapnikov, Phys. Rev. A 66, 013606 (2002).

[20] M. Baranov, Ł. Dobrek, K. Góral, L. Santos, and M. Lewenstein, Phys. Scr. T201, 74 (2002).

[21] M. Amoruso, I. Meccoli, A. Minguzzi, and M.P. Tosi, Eur. Phys. J. D 7, 441 (1999).

[22] G.M. Bruun and C.W. Clark, Phys. Rev. Lett. 83, 5415 (1999).

[23] A. Csordás and R. Graham, Phys. Rev. A 63, 013606 (2001).

[24] B. DeMarco and D.S. Jin, Phys. Rev. Lett. 88, 040405 (2002).

[25] L. Vichi and S. Stringari, Phys. Rev. A 60, 4734 (1999).

[26] V.M. Pérez-García, H. Michinel, J.I. Cirac, M. Lewenstein, and P. Zoller, Phys. Rev. Lett. 77, 5320 (1996).

[27] S. Yi and L. You, Phys. Rev. A 63, 053607 (2001).

[28] K. Góral and L. Santos, Phys. Rev. A 66, 023613 (2002).

[29] E. Zaremba and H.C. Tso, Phys. Rev. B 49, 8147 (1994). 